\title{
TRANSMISSION AND RECEPTION OF DATA THROUGH USB USING FPGA
}

\author{
Dinah Ann Varughese ${ }^{1}$ \\ ${ }^{1}$ Assistant Professor, ECE Department, St. Thomas College Of Engineering \& Technology, Kerala, India.
}

\begin{abstract}
The Universal Serial Bus (USB) replaces different communication interfaces with high speed of data transfer rate. To enable high speed data transfer, I proposed FPGA based controller for the transmission and reception of data. This FPGA based controller should be designed in such a way that it must be able to detect and communicate with USB port and must be able to transmit large amounts of data between a personal computer and a memory storage device.
\end{abstract}

$* * *$

\section{INTRODUCTION}

The universal serial bus (USB) is an I/O interface standard. It is a connection between a host computer and a number of peripheral devices. And it was originally created to replace a wide range of slow and different buses-the parallel and the serial. USB has grown beyond these slow connections and supports almost every type of device that can be connected to a PC. Features of USB are high-bandwidth, easiness of use, hotplug capability. Besides, the driver is one of the important technological aspects in the development of USB device, which directly affects the system performance of entire equipment, so the writing of driver is necessary inorder to realize the software's access to hardware.

In this project paper it is proposed to implement an USB controller which provides data transfer between an USB device and PC. It is implemented using FPGA integrated circuit- Xilinx Spartan 3E.

\section{SYSTEM ARCHITECTURE}

\subsection{Architecture Overview}

The basic idea of this proposed project is to implement a hardware of a USB controller based on FPGA. The core of a USB storage device will be a bulk data transfer type capable of large amount of data transfer from a memory to another memory. The USB core schematic is shown below:

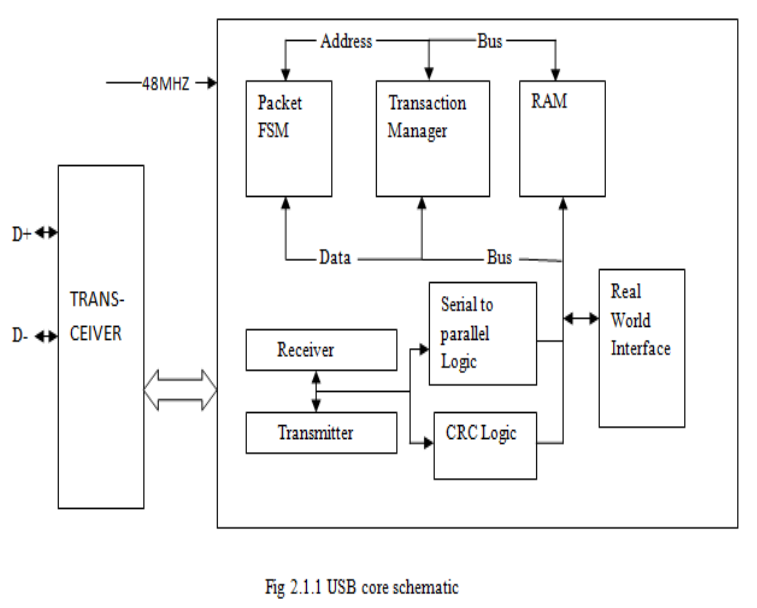

For the development of this proposed core, certain sequence of steps should be carried out. They are as follows:

\subsubsection{USB Protocol}

It is used for USB protocol decoding and information read out. The objective of this stage is to assure the correct decoding of data packets. A transceiver integrated circuit USB1T11A is used to interface the USB lines. Once the USB bus data is received in NRZI format, a decoding process takes place. Decoding process is done in VHDL. A NRZI to RZ converter circuit is used for detecting the data being received. As data is received the cyclic redundancy check (CRC) logic is performed inorder to validate the received data.

\subsubsection{USB Transmitter}

The USB transmitter manages the data transmission output i.e. the addition of a zero bit in case six consecutive logic ones are transmitted (bit stuffing). 


\subsubsection{External Interface}

External interface can be made through a PicoBlaze microcontroller.

\subsection{Objective}

The main objective of this paper is to implement a FPGA based core in order to receive and transmit the data from a host device (PC) to USB device or vice-versa.

\section{SYSTEM DESIGN REQUIREMENTS}

\subsection{Functional Requirement Specification}

- Since this project deals with the data transferring between two memory storage devices using bulk transfer type, the input and output of the proposed core must be data in the form of packets whose size cannot be exceed than the allowable size which is 512 bytes.

- $\quad$ Shift register is used for the serial to parallel logic. Out of the four shift registers, SIPO shift register is taken.

- Clock to the core is provided by the internal clock of the hardware tool used.

\subsection{Hardware and Software Realization}

The proposed design is planned to be described using VHDL(VHSIC Hardware Description Language) and simulated by ModelSim and can be implemented on Xilinx Spartan 3E FPGA(Field Programmable Gate Array).

\subsection{Hardware Implementation}

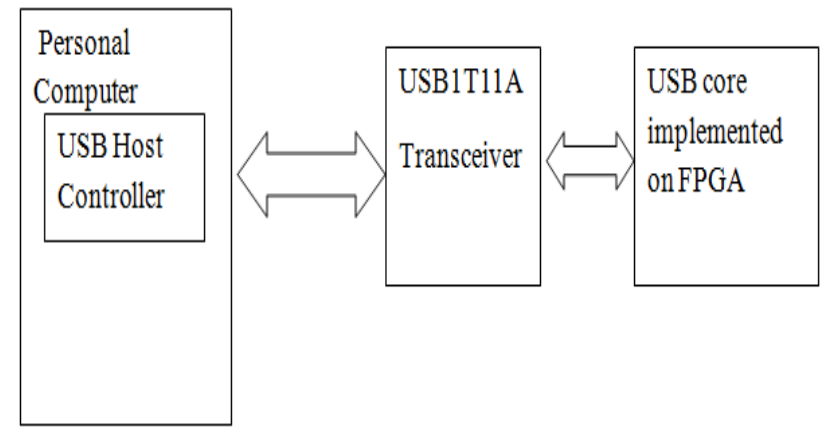

Fig 2 Hardware Model

This represents the actual transfer of data across the USB cable between the host system (PC) and the USB devices. The host side consists of the USB host controller, while the USB side contains the USB interface within the device. All communication on USB originates at the host under software control. The host hardware contains the USB host controller, which initiates transactions over the USB system.

\section{IMPLEMENTATION METHODOLOGY SIMULATION}

\subsection{Introduction}

This chapter describes the implementation of the proposed controller. It consists of transmitter section, receiver section and a controller section. The controller section controls the transmission and reception of the data. The controller section does the NRZI encoding, bit stuffing for the transmitter section only when the control transmits signal is high. The controller section does the NRZI decoding, bit unstuffing, CRC generation, CRC checking for the receiver section only when the control receive signal is high. The controller section extracts the required data from a continuous serial data stream and stores it in a buffer which is placed in a receiver section. The proposed core is successfully simulated in ModelSim.

\subsection{Implementation of Receiver Section}

\subsubsection{NRZI Decoder}

The USB devices employ NRZI data encoding for transmission of data. In NRZI encoding, logic 1 is represented by no change in level and logic 0 is represented by a change in level. So there is need for conversion of NRZI to RZ.

\subsubsection{Bit Unstuffer}

In order to ensure adequate signal transitions, bit stuffing is employed by the transmitting device when sending a packet on USB. A zero is inserted after every six consecutive ones in the data stream before the data is NRZI encoded, to force a transition in the NRZI data stream.

\subsubsection{Byte Counter}

It counts the number of bit received which helps to identify the amount of data.

\subsubsection{CRC Checker}

The Cyclic Redundancy Checksums (CRC) is used to protect all non-PID fields in token and data packets from errors.

\subsection{Implementation of Transmitter Section}

\subsubsection{NRZI Encoder}

The USB devices employ NRZI data encoding for transmission of data packet. In NRZI encoding, logic 1 is represented by no change in level and logic 0 is represented by a change in level.

\subsubsection{Bit Stuffer}

It is employed by the transmitting device when sending a packet on USB. A zero is inserted after every six consecutive ones in the data stream before the data is NRZI encoded, to 
force a transition in the NRZI data stream. This bit stuffer adds the stuff bit if six consecutive ones are found.

\subsubsection{Serial Shifter}

It is a serial shift register. It converts the parallel data to serial data stream.

\subsubsection{Byte Counter}

This byte counter counts the number of bytes that are transmitted.

\subsection{Functional Requirements}

The proposed core needs software and hardware requirements. The core is simulated using ModelSim and written in VHDL code. The core is implemented on Xilinx FPGA.

\subsection{Simulation Results}

\subsubsection{Transmitter Section}
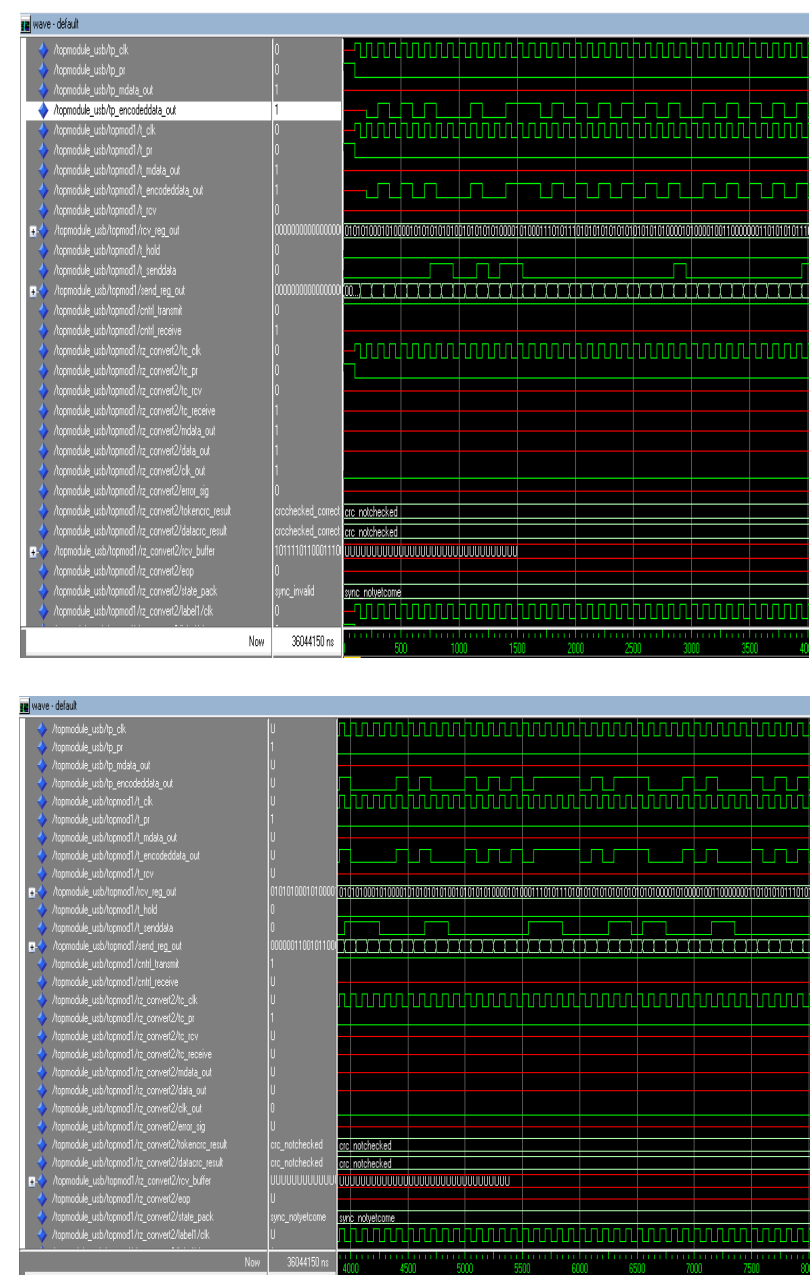
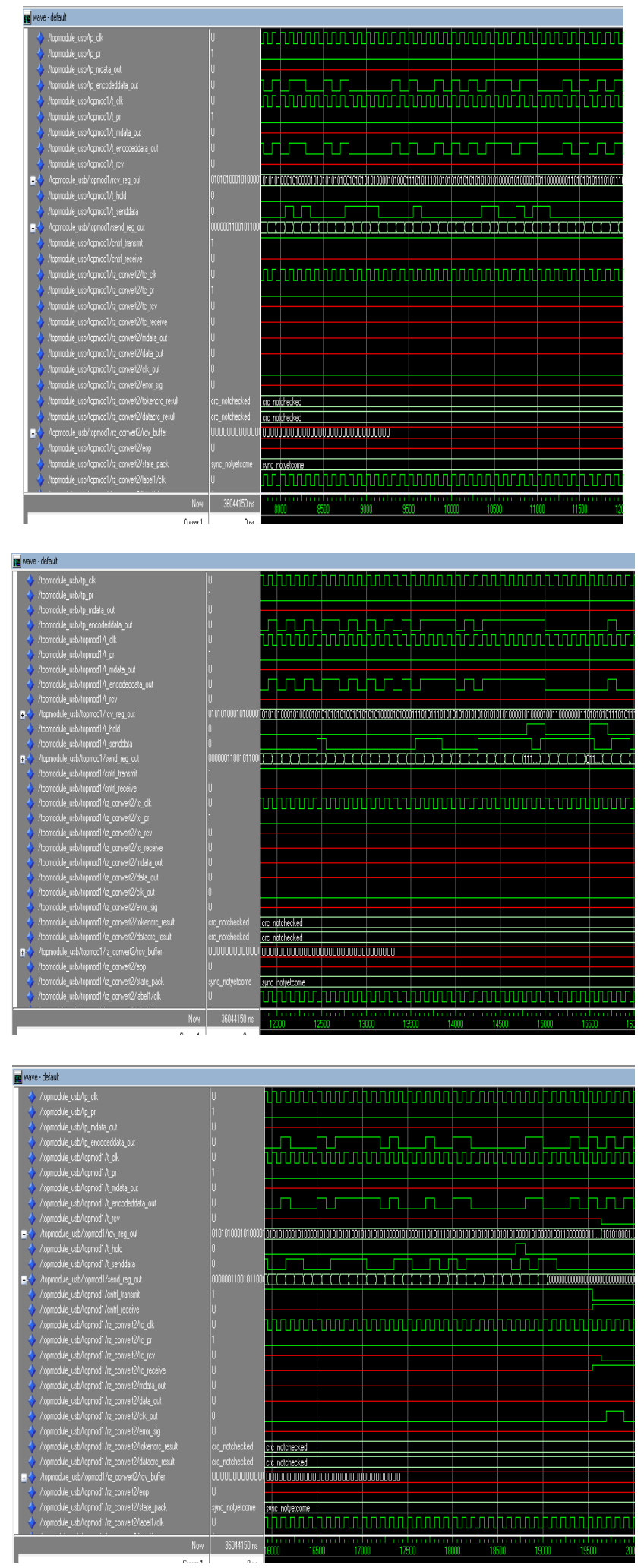

Fig 3. Simulation Results of Transmitter Section 


\subsubsection{Receiver Section}
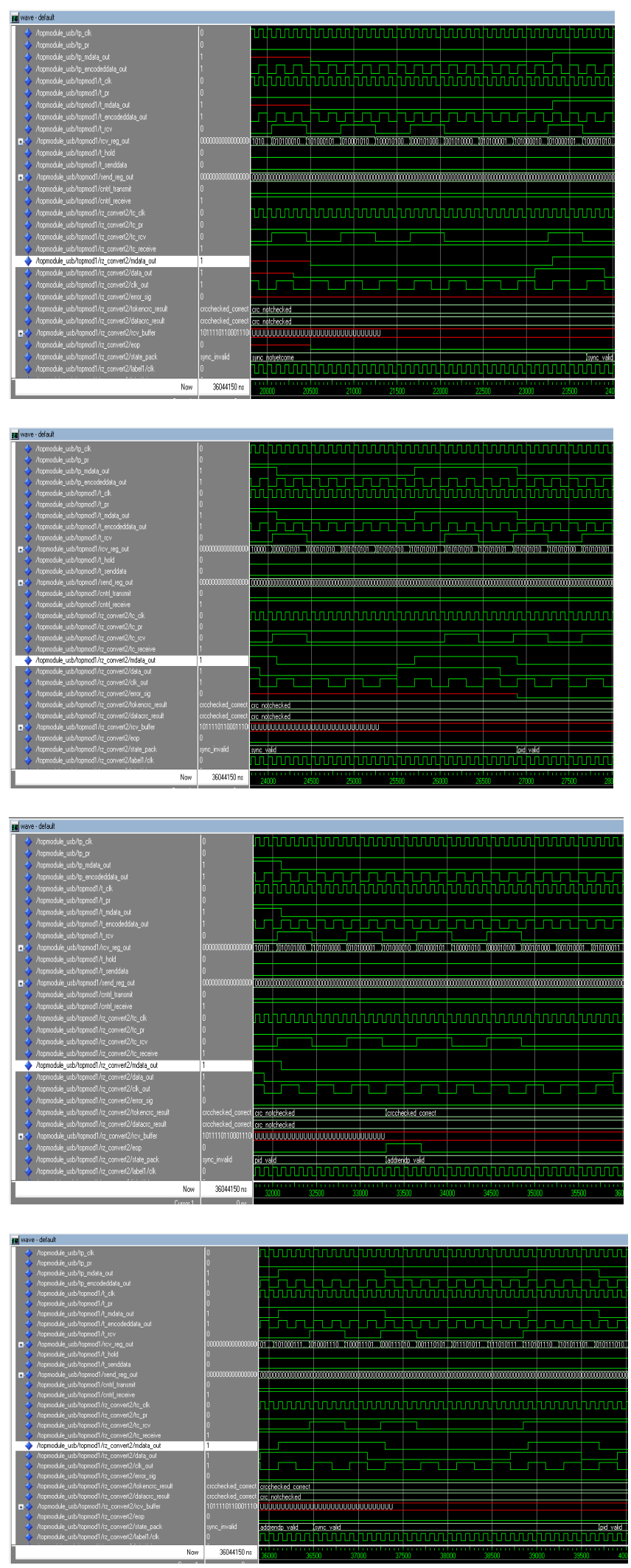
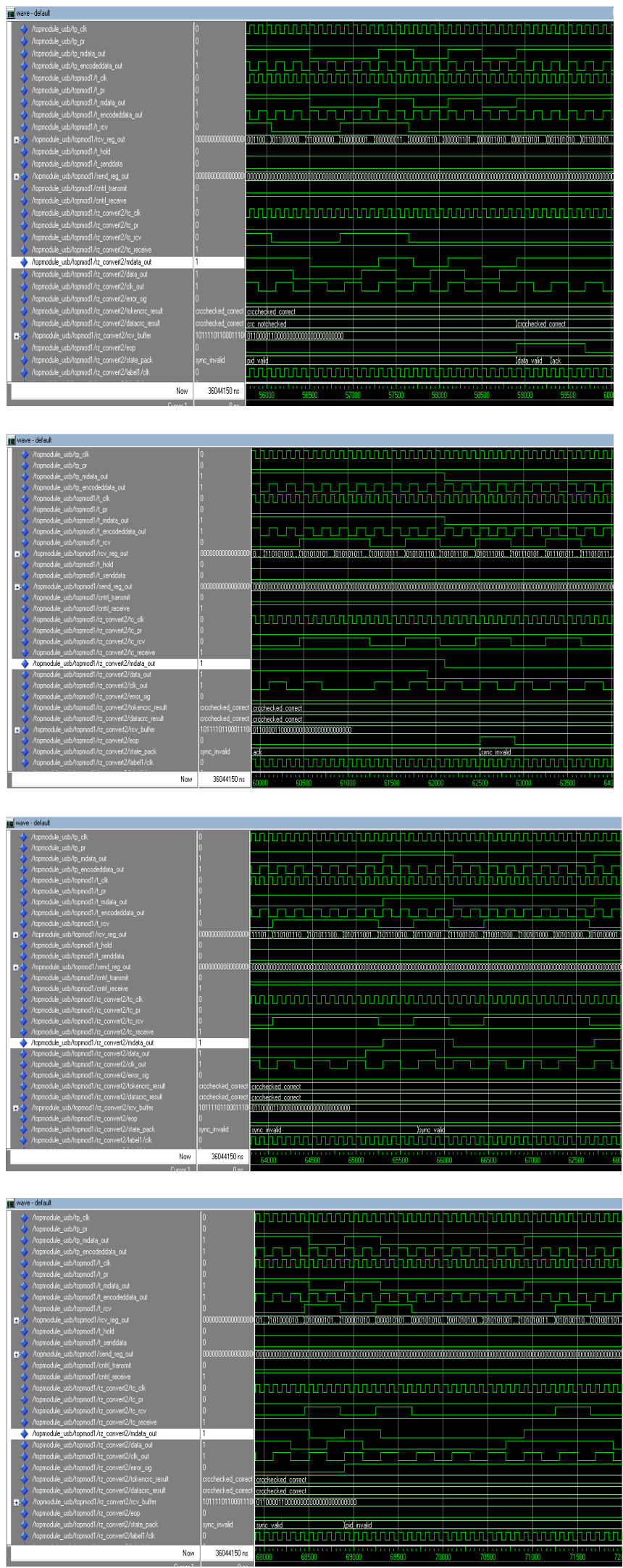

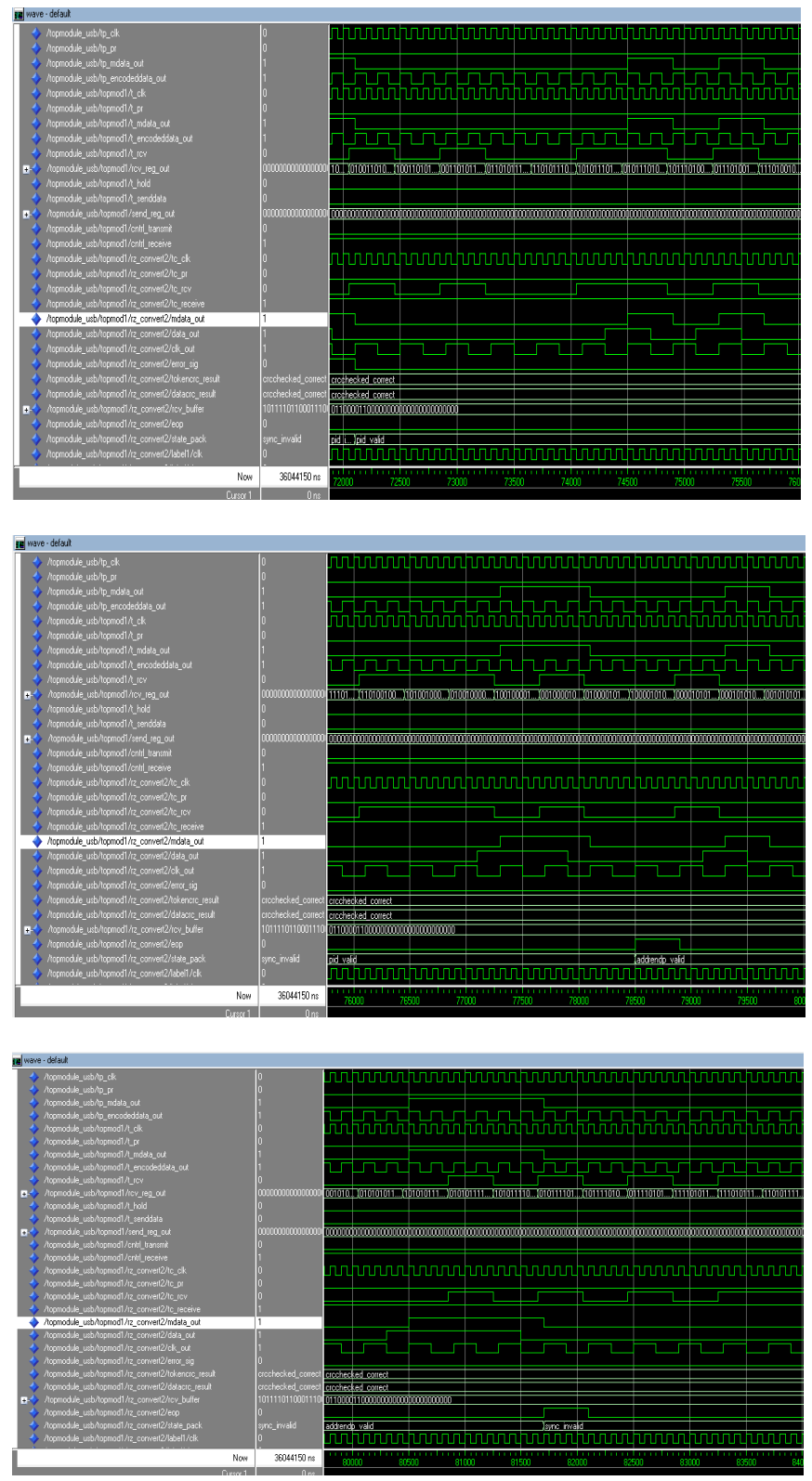

Fig 4 Simulation Results of Receiver Section

\subsection{Design Summary}

Table 1. Device Utilization Summary

\begin{tabular}{|l|r|r|r|r|}
\hline Logic Utilization & Used & Available & Utilization & Note(s) \\
\hline Number of Slices: & 461 & 3584 & $12 \%$ & \\
\hline Number of Slice Flip Flops: & 236 & 7168 & $3 \%$ & \\
\hline Number of 4 input LUTs: & 746 & 7168 & $10 \%$ & \\
\hline Number of bonded lOBs: & 12 & 173 & $6 \%$ & \\
\hline Number of GCLKs: & 3 & 8 & $37 \%$ & \\
\hline
\end{tabular}

\section{CONCLUSIONS}

This paper is an approach for the implementation of the USB device core on FPGA. The proposed core can also be used in a memory storage device using bulk data transfer type. The currently available memory storage device contains a flash memory and a controller part. It requires both hardware and software. Hence the memory for a given specification of a storage device is less compared to the proposed core. Since the proposed core is implemented using hardware, more memory is available and increases the speed of the data transfer.

To conclude the proposed core has a number of important advantages as the small space it requires from the FPGA circuit, low cost and the advantage of using internal resources included in the selected FPGA, as Pico Blaze microcontroller, included in Xilinx Spartan III series and that is part of the USB core.

\section{REFERENCES}

[1]. Universal Serial Bus Specification. September 1998. www.usb.org

[2]. USB IT1 1A Universal Serial Bus Transceiver data Sheet. Fairchild Corp. March 2005.

[3]. Fairchild Corp. Application Note 5015 - USB Transceiver and Specification Compliance.

[4]. Penny Orwick - Guy Smith. "Developing Drivers with the Windows Driver Foundation." Microsoft Press. April 2007. ISBN-13: 978-0735623743

[5]. Walter Oney. "Programming the Microsoft Windows Driver Model", Second Edition. Microsoft Press, December 2002. ISBN-13: 978-0735618039

[6]. Don Anderson, "Universal Serial Bus System Architecture", 2nd. edition, MindShare Inc. 2001. ISBN-13: 978-0201309751

[7]. Jan Axelson, "USB Complete-The Developer's Guide", 4th.edition, Lakeview Research LLC, 5310 Chinook Ln., Madison WI 53704. ISBN-13 978-1-931448-08-6

\section{BIOGRAPHIE:}

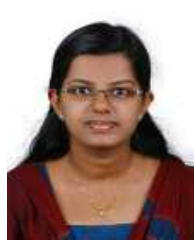

Dinah Ann Varughese was born in Chengannur,India in 1987. She received the BTech degree in Electronics and communication Engineering from Mahatma Gandhi University, India in 2009, and the MTech in VLSI and Embedded Systems from Cochin University of Science and Technology, Cochin in 2011. In 2011, she joined the department of Electronics and Communication Engineering, St.Thomas College of Engineering and Technology Chengannur as Assistant Professor. Her research interest is centered mainly in designing of controller part using FPGA, Microcontroller based system design. 